\title{
Performance study on the effect of filter curve in CWDM System for the access network
}

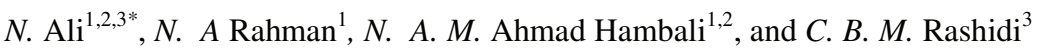 \\ ${ }^{1}$ School of Microelectronics Engineering, Universiti Malaysia Perlis (UniMAP), 02600 Arau, Perlis, Malaysia \\ ${ }^{2}$ Semiconductor Photonics \& Integrated Lightwave Systems (SPILS), School of Microelectronic Engineering, Universiti Malaysia \\ Perlis, Pauh Putra Main Campus, 02600 Arau, Perlis, Malaysia \\ ${ }^{3}$ Advanced Communication Engineering, Centre of Excellence-School of Computer and Communication Engineering, Universiti \\ Malaysia Perlis
}

\begin{abstract}
This paper presents the study on the effect of filter variation on the coarse wavelength division multiplexing (CWDM) system. The filter curve will affect the performance of the CWDM system due to changes of received power lever and isolation of the signal. The significant impact on the received power level and isolation can be found when the required signal is isolated from unwanted signal by the steep curve of filter. As a result, BER of $1.0 \times 10^{-12}$ was obtained corresponding to receive power level of -24.27 $\mathrm{dBm}$ with isolation of $23.22 \mathrm{~dB}$. When the wavelength spacing is reduced to $1 \mathrm{~nm}$, the isolation is only 11.30 $\mathrm{dB}$ and $\mathrm{BER}$ increased to $5.49 \times 10^{-7}$ with a received power of $-15.39 \mathrm{dBm}$.
\end{abstract}

\section{Introduction}

Internet growth and expansion has becoming dense in access network $[1,7]$. The migration of copper to optical network is already an obsolete solution. The access network is not for long haul transmission system, which loss in terms of power penalty will not contribute an impact to data quality during transmission. The main consideration is the cost of the system because the optical network is expensive [2,8]. Utilizing previous installed network can save cost in optical system. The CWDM system gives opportunity for the existing network installed to be fully utilized. It is considered as a cost effective option which more network can be installed in one transmission system and the cost of the equipments i.e., multiplexer and filter is economical compare to other WDM system[3]. The filter used in the CWDM system is normally wide bandwidth where the price is less. The filter is not required to be narrow due to the wider wavelength spacing between channels. The wide filter shape also prevent lost due to the wavelength shifting occur during transmission. The isolation of the signal is determined by the filter shape. Filter with steeper shape produces higher isolation. The spacing of the channel also can reduce the isolation level if the spacing between the channels is small.
For CWDM, spacing is larger and so is the filter bandwidth. Hence, inter-channel crosstalk is unlikely to happen in this system. Isolate the adjacent signal will also reduce the received power and the transmission distance as well. Therefore, it is important to study the filter effect on performance of the access network for CWDM system. In this paper, the received power level and isolation of the signal is investigated where the variation of these parameters contribute by the filter curve. The shape of the filter in the system setup (refer to Figure 1) is the main contributor to the isolation and the received power level of the system. These parameters will contribute on the performance of the system which BER of the system is investigated due to changes of isolation and received power level.

In this paper, testing was done using standard SDH data rate at $2.5 \mathrm{Gbps}$. The parameters under study were received power and isolation with respect to filter's curve

\section{Experiment Setup}

The experiment setup to analyze the performance of the new CWDM system is shown in Figure 1.

Corresponding author: norshamsuri@unimap.edu.my 


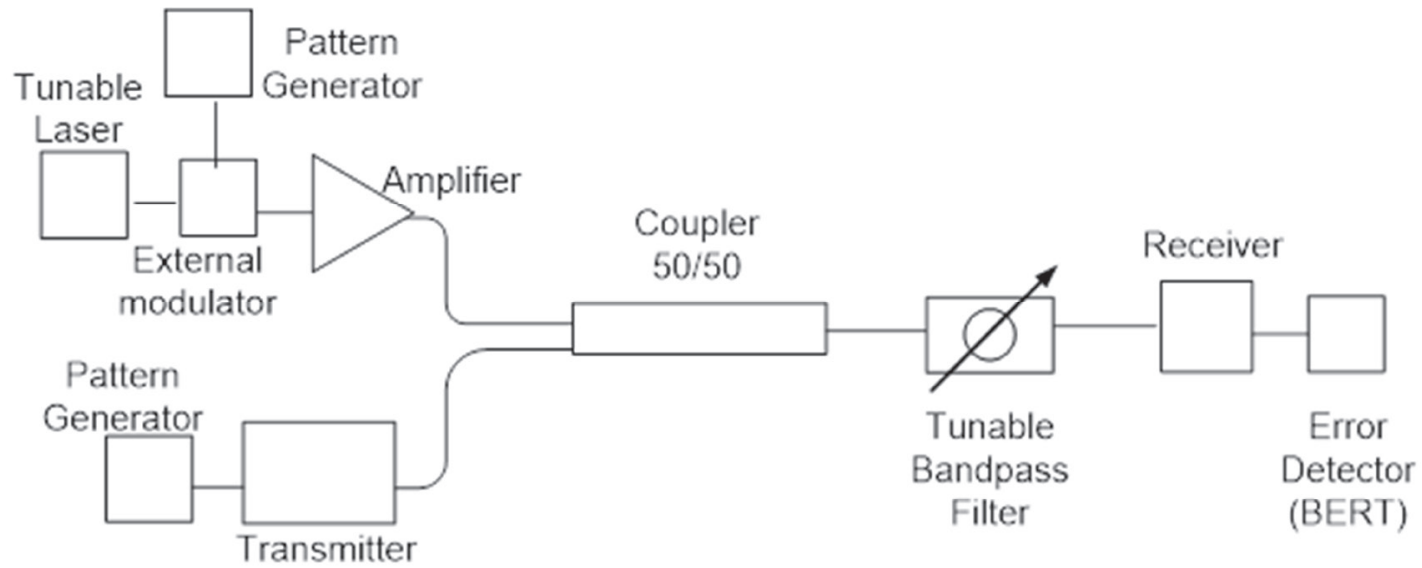

Fig. 1. Experiment setup

This setup is a point to point transmission where the distance of the transmission is based on power budget calculation. The main blocks of the experiment are the transmitter, coupler, tuneable bandpass filter and the receiver. Coupler acts as the multiplexer while the tuneable bandpass filter acts as the de-multiplexer. The main signal is modulated by the external modulator, while the adjacent signal is modulated from the $2.5 \mathrm{Gbps}$ transmitter. The main signal has to be amplified since the external modulator attenuates the transmitted power by $8.3 \mathrm{~dB}$. Both signals are then, multiplexed by coupler before being filtered by the tuneable bandpass filter to recover back the main signal. The performance of this setup is determined by the BER value detected by BER tester at the receiving side. The BER calculation is based on the $95 \%$ confidence level at 1e-12 error rates with 2 minutes requirement for every data collected.

\section{Result and Discussion}

Figure 2 below shows the spectrum of the filter where the $3 \mathrm{~dB}$ bandwidth of the filter is approximately $1.5 \mathrm{~nm}$. The curve of the filter is shown to be steeper at the upper side of spectrum and less steep at the leg of the spectrum. From the graph, the insertion loss of the filter can be determined by investigating the spectrum or the shape of filter. The changes of the receive power level is depending on the form of the filter shape. In order to isolate the adjacent signal, the filter will be adjusted to the minimum power level of adjacent signal

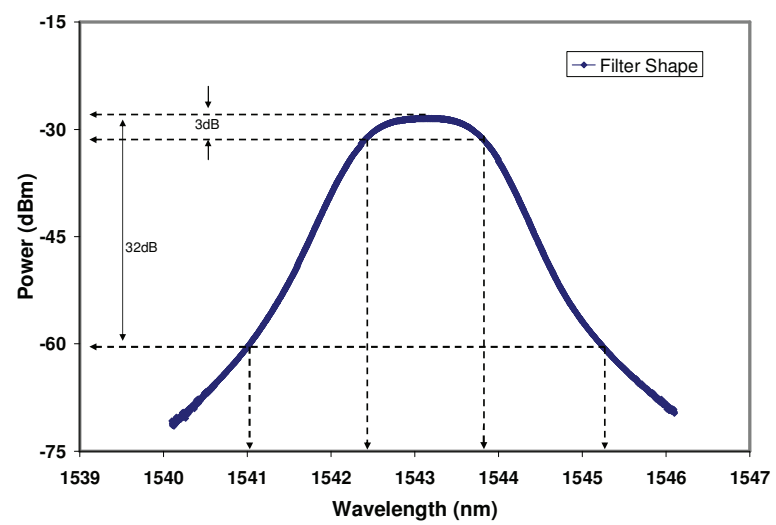

Fig. 2. Broad filter curve

To this extend, the character of the filter slope contribute most to the changes in the received power level. The isolation of signal is more effective when the isolation is done between the $3 \mathrm{~dB}$ points of the curve (at $-35 \mathrm{dBm}$ as shown in Figure 2) and at $31 \mathrm{~dB}$ point (at $-60 \mathrm{dBm}$ as shown in Figure 1) of the curve. This is the range where the curve of the filter is steeper and the isolation of adjacent signal will not reduce the power level of received signal, hence higher isolation value can be achieved. The system is tested using $2.5 \mathrm{Gbps}$ data rate to check the system stability which introduces the broad filter. Normally the access networks support data rates up to $622 \mathrm{Mbps}$.

From the study, the new design provides up to 1.6 $\mathrm{nm}$ wavelength spacing as compared to normal CWDM system with minimum spacing of $20 \mathrm{~nm}$ [4]. The effect of filter's curve to the system is shown in Figure 3. The graph shows that when the wavelength spacing is reduced, the performance of the system is also reduced as shown by the increase of BER. 


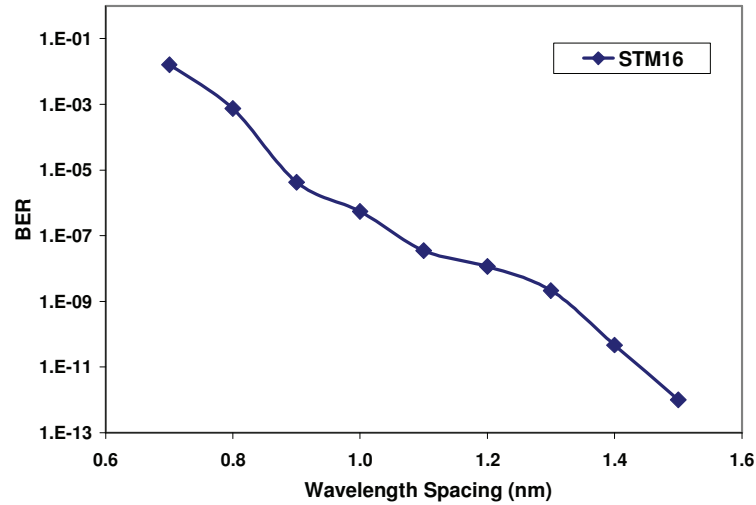

Fig. 3. Wavelength spacing effect on the performance of BER

The receive power level and the isolation of the signal is considered as the main design parameters because these are the main performance indicators for any optical receiver. Figure 4 presents the performance of the CWDM system when the isolation of the signal is reduced. From the graph, the minimum isolation that can be detected by the receiver with the least acceptable BER $(\approx 10-11)$ [5] any errors is $17.24 \mathrm{~dB}$. However, this data should be verified with the total received power since both of these parameters are interrelated. The isolation of the signal can be smaller than $17.24 \mathrm{~dB}$ but the received power level should be increased in order to maintain the data quality. The reason is that, the receiver has minimum sensitivity level, if the received power is lower than the sensitivity value, the receiver cannot distinguish between the actual signal or noises, even if the isolation of the signal is still acceptable.

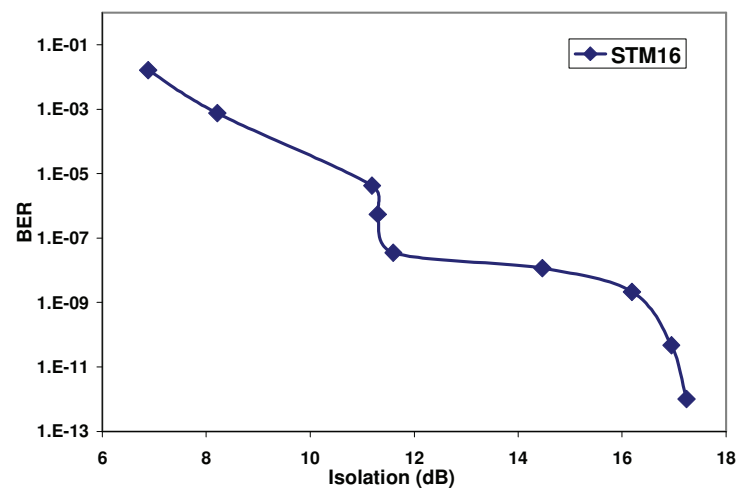

Fig. 4. The performance of isolation adjacent signal with respect to BER

On the other hand, the received power level can also be as small as $-10.41 \mathrm{dBm}$ as shown in Figure 5. In order to maintain the signal quality, the isolation of the signal should be bigger to ensure the receiver detects the signal correctly. These values are affected by the filter shape, where the steeper the shape of the filter curve, the better the quality of signal received [6]. Therefore, different type and characteristic of filter will give different value of isolation and receive power level and, it hence produced a different quality of performance.

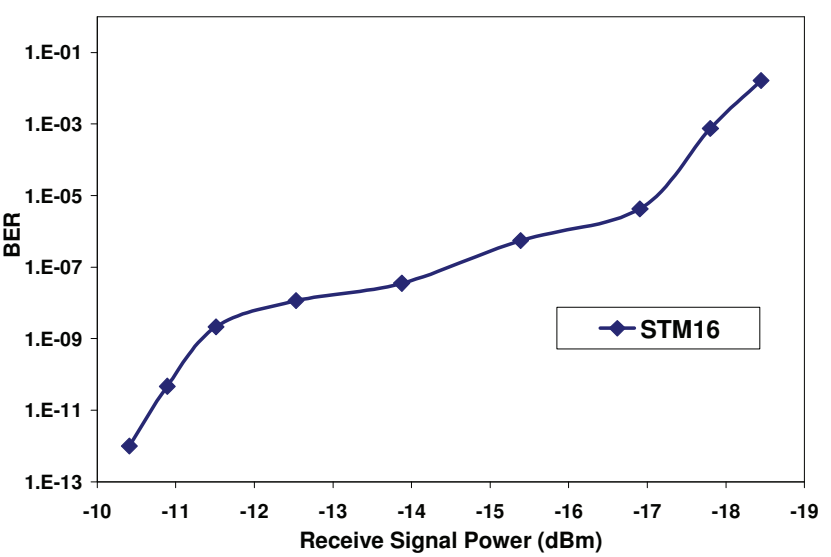

Fig. 5. The BER performance affected by the total received power.

Figure 6 shows the distance that can be establish by this new design CWDM system. The new design can reach up to $40 \mathrm{~km}$ with acceptable BER performance of the system compared to the normal CWDM system which only support up to $10 \mathrm{~km}$; this is improvement of $400 \%$ distance covered. This is done based on power transmitted around $-7 \mathrm{dBm}$ with power margin of $13 \mathrm{~dB}$. The other advantage of this new design it utilizes wavelength spacing of $1.6 \mathrm{~nm}$ as compared to the normal design at distance of $40 \mathrm{~km}$. By implementing this new system, the cost for the each link can be reduced and the load of the link can be increased in order to support the high capacity access network.

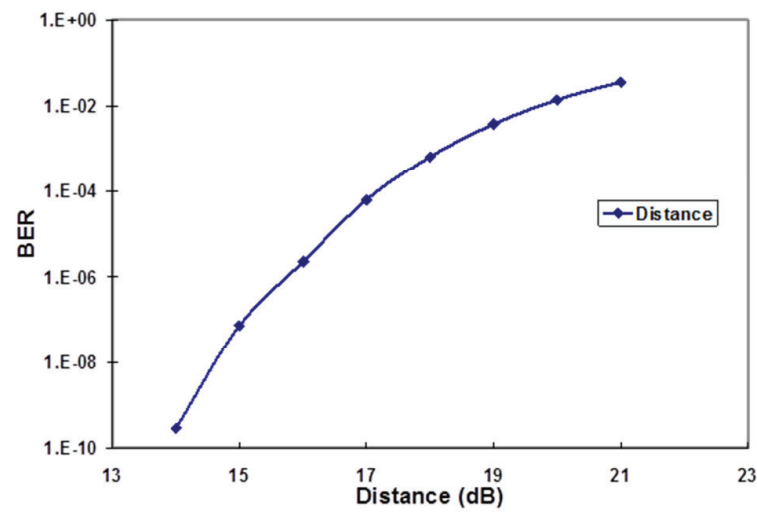

Fig. 6. Distance of transmission system due to BER performance 


\section{Conclusion}

The study demonstrates the effect of filter curve on the performance of the new CWDM system on the access network. The new system can achieved a distance of 40 $\mathrm{km}$ at $1.6 \mathrm{~nm}$ spacing with data rate of $2.5 \mathrm{Gbps}$. Furthermore, the new design optimizes the channel spacing, and the filter curves until it gives a better performance signal to the existing WDM system. As a result BER of $1.0 \times 10^{-12}$ was obtained corresponding to receive power level of $-24.27 \mathrm{dBm}$ with isolation of $23.22 \mathrm{~dB}$. When the wavelength spacing is reduced to 1 $\mathrm{nm}$, the isolation is only $11.30 \mathrm{~dB}$ and BER increased to $5.49 \times 10^{-7}$ with a received power of $-15.39 \mathrm{dBm}$.

The author would like to acknowledge this work was supported by the High Ministry of Education Malaysia. The work has been done in 2003 and yet not been published. The author would like to thanks to Late Prof Dr Mohamed Khazani Abdullah and his team at Universiti Putra Malaysia previously in 2003. Most of the work was done in Universiti Putra Malaysia previously.

\section{References}

1. Elnegaard K. N., Stordahl K., Ims L. A.; International Symposium on Services and Local access, ISSLS 2000, Stockholm, Sweden, 18-23 June 2000.F. De Lillo, F. Cecconi, G. Lacorata, A. Vulpiani, EPL, 84 (2008).

2. G. Held "Best Practice Series Network Design Principles and Applications", Auerbach Publications, Florida, (2000).

3. Roka, R.; Mobile Future and Symposium on Trends in Communications, 2003, SympoTIC '03. Joint First Workshop Oct. 2003, pp160 162.(2003).

4. RBN, "Characteristic of CWDM: Roots, Current status and Future Opportunity", White Paper, Redfren Broadband Networks, San Francisco,(2002).

5. Djafar K. Mynbaev, and Loell L. Scheiner.: "Fiber-Optic Communication Technology." Prentice-Hall, New Jersey, ( 2001).

6. Leo, C.J.; Ramana, P.V.; Sudharsanam, K.; Electronics Packaging Technology, 2003 5th Conference (EPTC 2003), pp 647-651, (2003).

7. Pastor-Satorras, Romualdo, Alexei Vázquez, and Alessandro Vespignani, Phys. Rev. Let. 87,25 (2001): 258701.

8. de Laat, Cees, Erik Radius, and Steven Wallace. Future Generation Computer Systems, 19,6 pp 999-1008, (2003). 